\title{
OPEM
}

www.opem.org

Oriental Pharmacy and Experimental Medicine 2008 7(5), 501-508

DOI 10.3742/OPEM.2008.7.5.501

\section{Analgesic effect of Clematidis Radix (CR) herb-acupuncture in a rat model of pain and inflammation}

\author{
Hye-Jeong Hwang ${ }^{1}$, Seung-Tae Kim ${ }^{1,2}$, Hye Jung Lee ${ }^{1,2}$, Young-Suk Kim ${ }^{3}$, In-Sop Shim ${ }^{4}$, Hi-Joon \\ Park $^{1,2}$, Won-Ju Choi ${ }^{5}$, Jang-Hyun Kim ${ }^{5}$ and Dae-Hyun Hahm ${ }^{1, *}$ \\ ${ }^{1}$ Acupuncture \& Meridian Science Research Center, Kyung-Hee University, Hoigi-dong, Dongdaemoon-gu, Seoul \\ 130-701; ${ }^{2}$ Department of Meridian \& Acupuncture, College of Oriental Medicine, Kyung-Hee University, Hoigi- \\ dong, Dongdaemoon-gu, Seoul 130-701; ${ }^{3}$ Department of Oriental Internal Medicine, College of Oriental \\ Medicine, Kyung-Hee University, Hoigi-dong, Dongdaemoon-gu, Seoul 130-701; ${ }^{4}$ Department of Integrative \\ Medicine, The Catholic University of Korea, Seoul, 137-701; ' Department of Oriental Medicine, Dongkuk \\ University, Kyeongsangbuk-do, 780-714, Korea
}

\begin{abstract}
SUMMARY
Anti-nociceptive and anti-inflammatory effects of Clematidis Radix (CR)-distillates were investigated in three different pain animal models. Highly purified distillate of CR was injected to Zusanli (ST36) acupoint, called CR herb-acupuncture in the Korean traditional medicine. In tail flick latency test, the CR herb-acupuncture treatment did not show a significant effect of relieving acute phasic pain. To investigate the anti-inflammatory effect of CR herb-acupuncture, inflammatory pain was induced by subcutaneous injection of formalin to the plantar tissue or intra-articular injection of carrageenan to the tibio-tarsal joint in the rats. And the medicinal effect of $\mathrm{CR}$ herb-acupuncture was evaluated by analyzing pain behavior such as licking or biting behavior, or by measuring weight distribution ratio between two foot and ankle circumference. In the rat formalin test, the analgesic effect of $\mathrm{CR}$ herb-acupuncture was more pronounced in the late phase (for $20 \mathrm{~min}$ after the early phase) than in the early phase (for the first $10 \mathrm{~min}$ post formalin injection). It also significantly alleviated the carrageenan-induced monoarthritis, in terms of weight distribution ratio and ankle edema. These results revealed that $C R$ herb-acupuncture was effective to treat the inflammatory pain and could be used as an analgesic treatment with an antiinflammatory effect.
\end{abstract}

Key words: Pain; Inflammation; Clematidis Radix; Herb-acupuncture; Rat

\section{INTRODUCTION}

Clematis root (Radix Clematidis in Latin and Wei Ling Xian in China) is a root of Clematis chinesis Osbeck, which contains anemonin, anemonol,

\footnotetext{
*Correspondence: Dae-Hyun Hahm, Acupuncture \& Meridian Science Research Center, Kyung-Hee University, 1 Hoigi-dong, Dongdaemoon-gu, Seoul 130-701, Korea. Tel: +8229610366; Fax: +8229632175; E-mail: dhhahm@ khu.ac.kr
}

sterol, saccharides, saponin, lacton, phenols and several amino acids. In the Korean traditional medicine, it has been widely used for treating various kinds of chronic diseases such as rheumatoid arthritis, gouts, lower back and limb pain, strokes, headaches, urinal disorders, irregular menstruation, deeply lodged fish bones in the throat, tonsillitis, tetanus, beriberi, etc. (Ahn, 1998). Its property is pungent and salty in taste, warm in nature, and acting on urinary bladder channel. 
Traditionally, this herb has been known to have strong action of dispersing the exopathogens and cold, eliminating the wind-dampness, and clearing stasis in channels to relieve arthralgia, and also to have the effects of elimination phlegm and softening the fish bone. Especially, the herbal extracts have been mainly used as an analgesic and anti-inflammatory agents as a COX inhibitor (Li et al., 2003).

In many Asian countries, acupuncture is widely used as one of the most effective medicinal treatments, especially for the chronic diseases accompanying severe pain such as sprain, osteoarthritis and rheumatoid arthritis (Filshie and White, 1998; Stux et al., 1995). And there are various methods for stimulating an acupuncture point (acupoint) in acupuncture treatments. Although electrical or manual stimulation of an acupoint is the most popular types in acupuncture therapy (Kim et al., 2005), recently the use of herbacupuncture has a tendency to increase. For the past two decades, a certain group of physicians in oriental medicine have been reported that the acupoint stimulation by subcutaneous injections of distilled herbal extracts, referred to herb-acupuncture or pharmaco-acupuncture in Korea, produced highly enhanced medicinal effects, compared to single treatment of either acupuncture or herbal therapy (Kwon et al., 2001; Yeom et al., 2003; Kim et al., 2005). The herbal extracts, used in the herbacupuncture, are manufactured by repeated water distillation with water for extreme purity and only tiny amount of the herbal extracts is injected into an appropriate acupoint of the body (Lee et al., 2000). Accordingly, both medicinal benefits of acupuncture and herbal therapy can be attempted by a single treatment of herb-acupuncture. However there have been little systematic and scientific studies on the therapeutic effectiveness of herbacupuncture, as of yet.

The ST36 acupoint (Zusanli), selected for Clematidis Radix (CR) herb-acupuncture in this study, is one of the most effective acupoints with a wide range of medicinal applications, for example, spasmolytic and analgesic effects on the gastrointestinal tract, and homeostatic effect in endocrine and metabolism. It has been also used for the treatments of aching of the knee joints and legs in the Korean traditional medicine (Ross, 1995; Stux et al., 1995; Filshie and White, 1998; Berman et al., 1999; Kwon et al., 2001).

The aim of the present study is to investigate the antinociceptive and anti-inflammatory properties of the aqueous extracts (distillates) from CR on experimentally induced pain and acute inflammation in the rats.

\section{MATERIALS AND METHODS}

\section{Animals}

Male Sprague-Dawley rats, weighting 230 - $250 \mathrm{~g}$ were used in this experiment. All animals were purchased from Samtaco Animal Corp. (Kyungki do, Korea). They were kept under controlled environmental conditions $\left(20 \pm 2^{\circ} \mathrm{C}, 12 / 12 \mathrm{~h}\right.$ lightdark cycles with lights on at 7 a.m., free access to food and water) for at least 1 week prior to study. Food and water were available ad libitum.

\section{Preparation of Clematidis Radix}

The CR was purchased from an oriental drug store (Jungdo Inc., Seoul, Korea). The distilled water extracts of $\mathrm{CR}$ for herb-acupuncture treatment were manufactured in an ampoule by the Korean Institute of Herbal Acupuncture (Seoul, Korea). The manufacturing processes were sequentially boiling, evaporation, condensation, and sterilization steps, which were always under the quality control of assuring that there were no suspended particles detectable by visible spectrophotometer. The extraction was started with $200 \mathrm{~g}$ of dried CR and finally produced $200 \mathrm{ml}$ of CR distillates for herbacupuncture. By the regulations of the Korean Institute of Herbal Acupuncture, the $\mathrm{CR}$ distillates passed the germ-free, anti-histamine, and endotoxinfree tests; and thus they were accepted as an s.c. 
injection grade for humans.

\section{Acupuncture treatment}

Herb-acupuncture treatment of acupoint was administered into the right Zusanli (ST36), located at the anterior tibial muscle about $5 \mathrm{~mm}$ lower and lateral to the anterior tubercle of tibia, using a 30 -gauge syringe for insulin injection. The rats in CON group were treated with saline into the same acupoint. And the rats in non-acupoint treatment group were intramuscularly treated into an arbitrary non-acupoint, located near the midline on the back. $0.5 \mathrm{ml}$ insulin syringe with 30 -gauge $5 / 16$ $(8 \mathrm{~mm})$ needle (BD Medical Systems, NJ, USA) was used for CR or saline injection.

\section{Antinociceptive study: tail flick latency test}

Rats were randomly divided into three groups; normal group (NOR, $\mathrm{n}=8$ ), CR herb- acupuncture treatment into the acupoint group (ACU, $n=14$ ), $\mathrm{CR}$ herb-acupuncture treatment into the nonacupoint group (NON-ACU, $\mathrm{n}=14$ ). The tail of the rat was immersed ( $5.5 \mathrm{~cm}$ from the tip) in a water bath at $50^{\circ} \mathrm{C}$ and time taken (in seconds) to flick the tail by using a stop watch was determined. Experiments were performed at room temperature $\left(22 \pm 1^{\circ} \mathrm{C}\right)$. During the test, rats were lightly restrained in a holder. Baseline tail flick latency was defined as the mean of three determinations at $10 \mathrm{~min}$ intervals before herb-acupuncture treatment. Only those rats with the baseline latency within the range of 4-7 s were used for further studies. Following herb-acupuncture administration, tail flick latency was measured at 10 min intervals. The antinociceptive effect in this test is presented either as latency or calculated as percentage change of the tail flick latency from the baseline level according to the formula: percentage change $(\%)=[$ (post-drug latency - pre-drug latency)/pre-drug latency] $\times 100$.

\section{Anti-inflammatory study: rat formalin test}

Rats were randomly divided into three groups; saline-treated control group $(\mathrm{CON}, \mathrm{n}=10), \mathrm{CR}$ herb-acupuncture treatment into the acupoint group (ACU, $\mathrm{n}=8$ ), $\mathrm{CR}$ herb-acupuncture treatment into the non-acupoint group (NON-ACU, $\mathrm{n}=8$ ). Five min after acupuncture treatment, $50 \mu \mathrm{l}$ of $5 \%$ formalin was subcutaneously injected under the right hind-paw plantar surface with a 30-gauge syringe (BD Medical Systems, NJ, USA) for insulin injection. After the formalin injection, the rats were immediately placed on clear plastic chamber $(20 \mathrm{~cm} \times$ $20 \mathrm{~cm} \times 20 \mathrm{~cm}$ ) with a mirror to allow an unimpeded view of the animal's paws and recorded the behavior through the video camera system for $30 \mathrm{~min}$. After video-recording, two experienced researchers independently calculated the paw licking time in every $5 \mathrm{~min}$. The licking behavior means that the rats lick or bit the formalin-injected paw. The paw licking time was classified into two phases: early phase (0-10 min after formalin injection) and late phase (10 - $30 \mathrm{~min}$ after formalin injection). The rats were treated with the aqueous extract.

\section{Anti-inflammatory study: carrageenan-induced ankle arthritis}

Rats were randomly divided into three groups; saline-treated control group ( $\mathrm{CON}, \mathrm{n}=11)$, CR herbacupuncture treatment into the acupoint group (ACU, n=8), CR herb-acupuncture treatment into the non-acupoint group (NON-ACU, $n=10$ ). Inflammation was induced by injection of $0.7 \%$ of carrageenan lamda (Sigma, St. Louis, MP, USA) in $50 \mu \mathrm{l}$ of pyrogen-free saline into the right tibiotarsal joint. To estimate the level of pain in the arthritic ankle, the amount of weight bearing force of the foot and circumference difference (CD) were measured before injection and then at 2, 4, 6 and $8 \mathrm{~h}$. The animals were placed in a test box with slanted plank. The mechano-transducer (CASS Co., BCL-1L, CI-2001A, KOREA) were placed below the hind paw. The bearing force of each hind-paw was estimated at the average for five seconds. And the mean bearing force was calculated from the data obtained by these 4 times estimation: 
$\% \mathrm{WDR}=100 \times($ weight borne by ipsilateral paw $/$ total weight borne by both paws).

The circumference of right ankle was measured around the lower edge of lateral and medial malleolus with a scaled soft ruler with elasticity. The $C D$ at $0,2,4,6,8$ and $24 \mathrm{~h}$ was indicated as the ratio of differences between formalin-injected and normal ankle circumferences to circumference of normal ankle, using following equation:

$\%$ difference of ankle circumference $=100 \times$ (circumference post - circumference pre)

/ circumference pre

Circumference post: circumference at each time point after formalin injection

Circumference pre: circumference of normal ankle before formalin injection

One ankle joint was gently extended for 2 times respectively with an inter-test interval of $5 \mathrm{~s}$.

\section{Statistical analysis}

The data were presented as the mean \pm S.E.M. Paw licking time was calculated and analyzed by oneway ANOVA followed by the Tukey post hoc test for further confirmation. Weight distribution ratio (WDR), difference of ankle circumference and tail flick latency were calculated and analyzed by repeated ANOVA followed by the Tukey post hoc test for further confirmation. The criterion for statistical significance was considered to be $P<0.05$.

\section{RESULTS}

\section{Tail flick latency}

The nociceptive pain sensitivity of rats was measured with the tail flick test. The effect of CR herb-acupuncture is presented in Fig. 1. The mean baselines of tail flick latency in the three groups of rats were $5.61 \pm 0.23,5.63 \pm 0.24$ and $5.75 \pm 0.18 \mathrm{~s}$, respectively. There were no significant differences

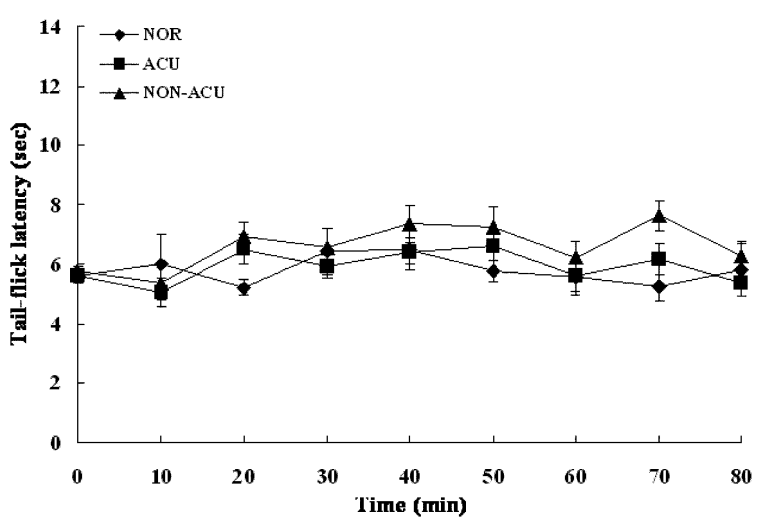

Fig. 1. Relationship between tail-flick latencies after immersion of the rats' tails in a 50 water bath.

$(P>0.05)$ among these data. Also, there was no significant increase in the reaction time with $C R$ herb-acupuncture in the tail flick test.

\section{Rat formalin test}

In order to investigate the analgesic effect of $C R$ herbal acupuncture, the rat formalin test was used as an acute inflammatory pain model and paw licking time was measured for assessing a pain behavior after the induction of formalin-induced pain. As shown in Fig. 2, paw licking time in each group in the early phase was as follows: CON, $111.13 \pm 7.90 \mathrm{~s} ; \mathrm{ACU}, 117.19 \pm 9.24 \mathrm{~s} ; \mathrm{NON}-\mathrm{ACU}$, $87.37 \pm 10.26 \mathrm{~s}$. In the case of late phase, paw licking

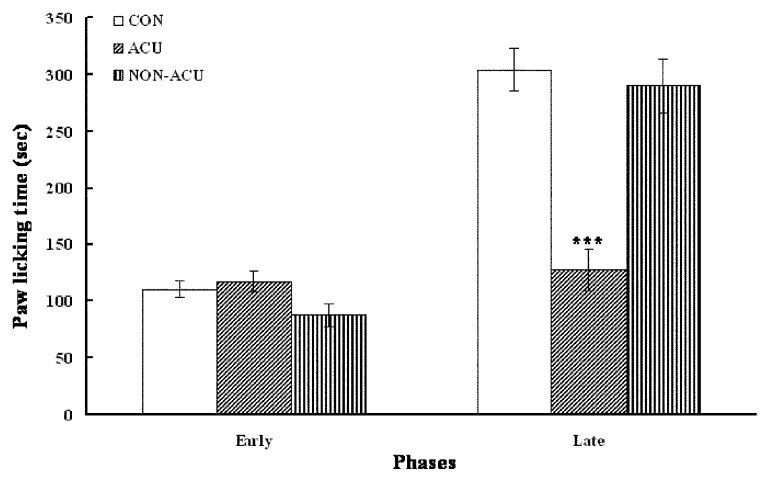

Fig. 2. Analgesic effect of $C R$ herb-acupuncture on formalin-induced pain behavior. Data were calculated and analyzed by one-way ANOVA followed by the Tukey post hoc test for further confirmation. ( ${ }^{x+k} P<$ 0.001 as compared to CON group) 
times were as follows: CON, $303.27 \pm 18.82 \mathrm{~s}$; ACU, $127.89 \pm 18.35 \mathrm{~s} ; \mathrm{NON}-\mathrm{ACU}, 289.61 \pm 23.48 \mathrm{~s}$. There were no significant differences between groups in the early phase ( 0 - $10 \mathrm{~min}$ after formalin injection). However, in the late phase (10 - 30 min after formalin injection), herb-acupuncture of $\mathrm{CR}$ reduced formalininduced pain behavior (paw licking time) than control group (CON). However, there were no significant differences between $\mathrm{CON}$ group and NON-ACU group in the late phase.

\section{Carrageenan-induced ankle arthritis}

\section{Weight distribution ratio}

In order to investigate the analgesic effect, the carrageenan-induced ankle arthritis was used as an acute inflammatory pain model and WDR as pain behavior was analyzed. As shown in Fig. 3, WDR (\%) before carrageenan injection was as follows: CON, $48.82 \pm 0.92 ; \mathrm{ACU}, 50.09 \pm 0.46$; NON-ACU, $48.09 \pm 1.43$. In $2,4,6,8,24 \mathrm{~h}$, WDR (\%) were as follows: CON, $42.13 \pm 1.92,33.41 \pm 2.25,19.03 \pm$ $3.08,15.39 \pm 3.13,30.19 \pm 3.13$; ACU, $46.37 \pm 2.70$, $31.81 \pm 3.63,30.71 \pm 3.59,27.20 \pm 4.04,38.56 \pm 3.33$; NON-ACU, $44.00 \pm 1.82,30.51 \pm 3.85,22.46 \pm 1.67$, $18.16 \pm 2.02,25.22 \pm 2.83$. Before the carrageenan injection $(0 \mathrm{~h})$, the mean WDR did not differ among

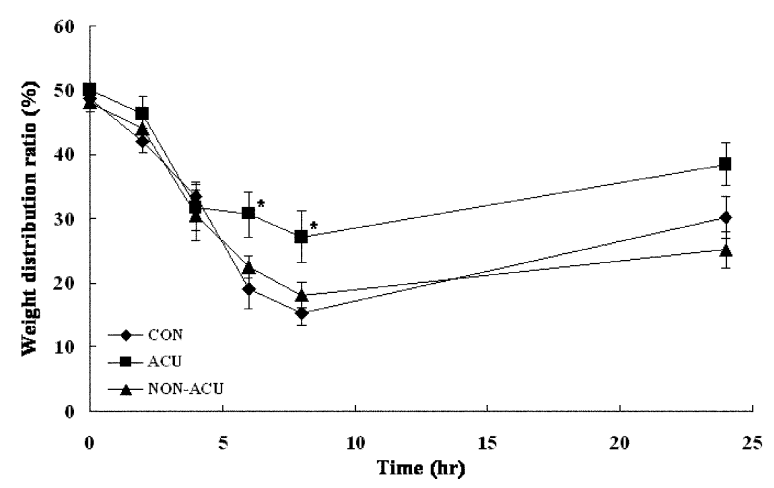

Fig. 3. Effect of CR-herb acupuncture on weight distribution between paws of carrageenan-induced ankle arthritic rats. Data were calculated and analyzed by repeated ANOVA followed by the Tukey post hoc test for further confirmation. ( $P<0.05$ as compared to CON group)

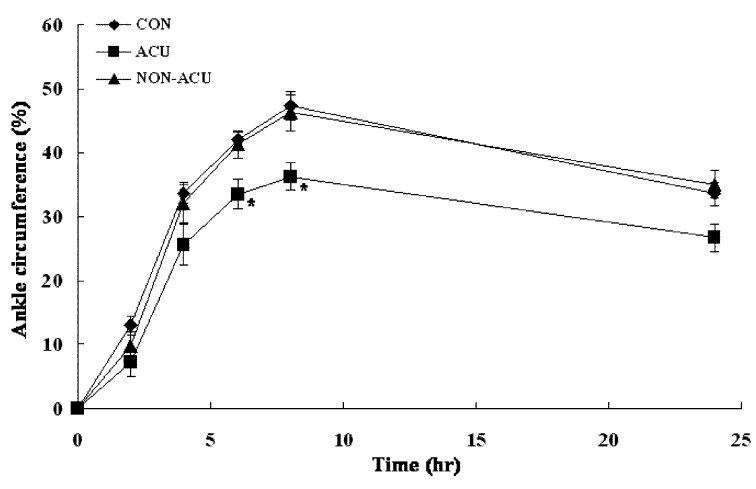

Fig. 4. Effect of CR-herb acupuncture on ankle circumferences of carrageenan-induced ankle arthritic rats. Data were calculated and analyzed by repeated ANOVA followed by the Tukey post hoc test for further confirmation. ( $P<0.05$ as compared to CON group)

the groups. The ratio in $\mathrm{CON}$ group was significantly decreased. The maximal ratio was measured at $8 \mathrm{~h}$. $\mathrm{ACU}$ group significantly differ from $\mathrm{CON}$ group at 6 and $8 \mathrm{~h}$.

\section{Ankle circumference difference}

The carrageenan-induced ankle edema in the rat was used to evaluate the anti-inflammatory effect of CR herb-acupuncture. As shown in Fig. 4, the ankle circumference before carrageenan injection $(0 \mathrm{~h})$ did not differ significantly among the groups. After arthritis induction, it was significantly increased, reaching a maximum at $8 \mathrm{~h}$. In the CON group, ankle circumference increased to $0,13.00 \pm$ $1.58,33.68 \pm 1.68,42.14 \pm 1.28,47.33 \pm 2.16,33.66 \pm$ 1.83 at $0,2,4,6,8,24 \mathrm{~h}$. In the ACU group, the increase rate of ankle circumference was 0, $7.19 \pm$ $2.22,25.65 \pm 3.13,33.46 \pm 2.35,36.30 \pm 2.14,26.74 \pm$ 2.13 and in the NON-ACU group, the increase rate of ankle circumference was $0,9.82 \pm 2.27,31.99 \pm$ $3.07,41.21 \pm 2.09,46.31 \pm 2.82,35.10 \pm 2.10$. Herbacupuncture of $C R$ significant inhibited the ankle edema than $\mathrm{CON}$ and NON-ACU groups. However, there were no significant differences between $\mathrm{CON}$ and NON-ACU groups. 


\section{DISCUSSION}

The results of this study show that the aqueous extract from $C R$ has an antinociceptive effect against the chemical stimuli provoked by the subplantar injection of formalin or by the injection of carrageenan into tibio-tarsal joint. However, this activity was very slight against thermal stimuli. This plant also presented an important antiinflammatory effect on acute edema.

In the rat formalin test, it is well established that the early $(0-10 \mathrm{~min})$ phase of formalin inducedpain behavior is produced by direct activation of primary afferent fibers, while pain behaviors associated with late (10 - $30 \mathrm{~min})$ phase are related to formalin-induced inflammatory reaction (Tjolsen et al., 1992). Therefore, this behavioral test can be used to verify the anti-nociceptive effect of a test compound and to investigate the functional mechanism. Several chemical mediators, such as histamine, kinin, serotonin and prostaglandins are released from damaged cells (Rosland et al., 1990). These mediators take part in the inflammatory response and are able to stimulate nociceptors and thus induce pain. Late phase pain may be blocked by drugs, which are known to reduce the inflammatory response (Hunskaar et al., 1986; Hunskaar and Hole, 1987; Shibata et al., 1989). In most cases, stimulation of pain endings in the periphery is chemical in origin. Excessive mechanical or thermal stimuli can obviously cause acute pain, but the persistence of such pain after the stimulus has been removed and the pain resulting from inflammatory or ischemic changes in tissues generally reflect a chemical stimulation of the pain afferents (Nikfar et al., 1997). Many studies have confirmed that inflammation takes place in the late phase (Rosland et al., 1990; Nikfar et al., 1997; Nikfar et al., 1998). From the results that the analgesic activity of CR herb-acupuncture was more pronounced in the late phase than in the early phase, it was suggested that the pain-relieving effect of CR herb-acupuncture might be resulted from the anti-inflammatory action.

In many animal studies, carrageenan has been used for inducing various types of inflammation, paw edema and acute mono-arthritis. It induces hyperalgesia and inflammation in rodents, which has been widely accepted as an appropriate model to examine the efficacy of anti-inflammatory and / or analgesic drug candidates. In previous reports, the injection of carrageenan into the ankle joint of one leg in rats produced edema and pain-related behaviors, and the WDR was used as one of the main tools to assess the severity of pain (Schött $e t$ al., 1994). Decreased WDR was one of the most commonly observed functional disabilities in mono-arthritic animals (Urban and Gebhart, 1999). In this study, the CR herb-acupuncture treatment into the ST36 acupoint significantly inhibited the WDR decrease in the arthritic rats, due to reducing the carrageenan-induced pain and inflammation. It suggested that CR herb-acupuncture could be a strong candidate of analgesic drug, controlling pain and inflammation.

The arthritic edema is a major symptom on arthritic patients (Bennell and Hinman, 2005), it usually resulted from the local action of multiple inflammatory mediators, including prostaglandins (Zhang et al., 1997), bradykinin (Wirth et al., 1992), NO (Handy and Moore, 1998), 5-hydroxytryptamine (Holsapple et al., 1980) and histamine (Stochla and Maslinski, 1982). Acupuncture reduced the severity of the edema by downregulating inflammatory substances such as prostaglandins, IL-1 $\beta$ (Fang et al., 2007) and TNF- $\alpha$ (Lee et al., 2004). So the inhibition of carrageenan-induced ankle edema in animals by the $C R$ herb-acupuncture treatment gave further support for proving its action as an anti-inflammatory drug.

In conclusion, $\mathrm{CR}$ herb-acupuncture significantly reduced formalin-induced pain behavior in the late phase of the rat formalin test, and alleviated pain and inflammation in carrageenan-induced ankle arthritis rat, in terms of weight distribution ratio and severity of the ankle edema. These results 
indicated that it was effective to treat the inflammatory pain and could be used as an analgesic treatment with an anti-inflammatory effect.

\section{ACKNOWLEDEMENTS}

This work was supported by the Korea Science and Engineering Faundation (KOSEF) grant funded by the Korea government (MEST) (R11-2005-014), Republic of Korea

\section{REFERENCES}

Ahn DK. (1998) Illustrated Book of Korean Medical Herbs, p. 304, Kyo-Hak publishing, Seoul.

Bennell K, Hinman R. (2005) Exercise as a treatment for osteoarthritis. Curr. Opin. Rheumatol. 17, 634-640.

Berman BM, Singh BB, Lao L, Langenberg P, Li H, Hadhazy V, Bareta J, Hochberg M. (1999) A randomized trial of acupuncture as an adjunctive therapy in osteoarthritis of the knee. Rheumatology $38,346-354$.

Fang JQ, Liu F, Shao XM, Wu YY. (2007) Effect of electroacupuncture on carrageenan-induced inflammation, IL-1beta and TNF-alpha concentrations and their mRNA expressions in toe tissue in rats. Zhen Ci Yan Jiu 32, 224-228.

Filshie J, White A. (1998) Medical Acupuncture-A Western Scientific Approach. 1st ed., pp. 341-360, Churchill Livingstone, London.

Handy RL, Moore PK. (1998) A comparison of the effects of L-NAME, 7- NI and L-NIL on carrageenaninduced hindpaw edema and NOS activity. $\mathrm{Br}$. J. Pharmacol. 123, 1119-1126.

Holsapple MP, Schnur M, Yim GK. (1980) Pharmacological modulation of edema mediated by prostaglandin, serotonin and histamine. Agents Actions 10, 368-373.

Hunskaar S, Berge OG, Hole K. (1986) Dissociation between antinociceptive and anti-inflammatory effects of acetylsalicylic acid and indomethacin in the formalin test. Pain 25, 125-132.

Hunskaar S, Hole K. (1987) The formalin test in mice: dissociation between inflammatory and noninflammatory pain. Pain 30, 103-114.

Kim HW, Kwon YB, Han HJ, Yang IS, Beitz AJ, Lee
JH. (2005) Antinociceptive mechanisms associated with diluted bee venom acupuncture (apipuncture) in the rat formalin test: involvement of descending adrenergic and serotonergic pathways. Pharmacol. Res. 51, 183-188.

Kwon YB, Lee JD, Lee HJ, Han HJ, Mar WC, Kang SK, Beitz AJ, Lee JH. (2001) Bee venom injection into an acupuncture point reduces arthritis associated edema and nociceptive responses. Pain 90, 271-280.

Lee JD, Kim SY, Kim TW, Lee SH, Yang HI, Lee DI, Lee YH. (2004) Anti-inflammatory effect of bee venom on type II collagen-induced arthritis. Am. J. Chin. Med. 32, 361-367.

Lee SK, Lee JD, Koh HK, Park DS, Lee YH, Kang SK. (2000) The study on the Hominis Placenta Aquaacupuncture solution. J. Korean Acupuncture Moxi. Soc. 17, 67-74.

Li RW, David Lin G, Myers SP, Leach DN. (2003) Anti-inflammatory activity of Chinese medicinal vine plants. J. Ethnopharmacol. 85, 61-67.

Nikfar S, Abdollahi M, Etemad F, Sharifzadeh M. (1997) Effects of sweetening agents on morphineinduced analgesia in mice by formalin test. Gen. Pharmacol. 29, 583-586.

Nikfar S, Abdollahi M, Sharifzadeh M, Eftekhar N. (1998) Interaction between lead acetate and morphine on antinociception in mice by formalin test. Gen. Pharmacol. 30, 489-493.

Rosland JH, Tjoisen A, Maehle B, Hole K. (1990) The formalin test in mice, effect of formalin concentration. Pain 42, 235-242.

Ross J. (1995) Acupuncture Point Cominations: the Key to Clinical Success. 1st ed., Churchill Livingstone, Edinburgh.

Schött E, Berge OG, Angeby-Möller K, Hammarstörm G, Dalsgaard CJ, Brodin E. (1994) Weight bearing as an objective measure of arthritic pain in the rat. J. Pharmacol. Toxicol. Methods 31, 79-83.

Shibata M, Ohkubo T, Takahashi H, Inoki R. (1989) Modified formalin test: characteristic biphasic pain response. Pain 38, 347-352.

Stochla K, Maslinski S. (1982) Carrageenan-induced oedema in the rat paw.-histamine participation. Agents Actions 12, 201-202.

Stux G, Berman B, Pomeranz B, Kofen P. (1995) Basics of acupuncture. 3rd ed., pp. 1-250, Springer-Verlag, Berlin. 
Urban MO, Gebhart GF. (1999) Supraspinal contributions to hyperalgesia. Proc. Natl. Acad. Sci. USA. 96, 7687-7692.

Wirth KJ, Alpermann HG, Satoh R, Inazu M. (1992) The bradykinin antagonist Hoe 140 inhibits carrageenan- and thermically induced paw oedema in rats. Agents Actions Suppl. 38, 428-431.

Yeom MJ, Lee HC, Kim GH, Shim I, Lee HJ, Hahm DH. (2003) Therapeutic effects of Hominis placenta injection into an acupuncture point on the inflammatory responses in subchondral bone region of adjuvantinduced polyarthritic rat. Biol. Pharm. Bull. 26, 14721477.

Zhang Y, Shaffer A, Portanova J, Seibert K, Isakson PC. (1997) Inhibition of cyclooxygenase-2 rapidly reverses inflammatory hyperalgesia and prostaglandin E2 production. J. Pharmacol. Exp. Ther. 283, 1069-1075. 